\title{
Endoscopic variceal ligation combined with sclerotherapy for management of gastroesophageal variceal bleeding in pediatric patients
}

\section{Ming-Ming Li}

Shanghai Jiaotong University School of Medicine

\section{Fang Sun}

Shanghai Jiaotong University School of Medicine

Man-Xiu Huai

Shanghai Jiaotong University School of Medicine

\section{Chun-Ying Qu}

Shanghai Jiaotong University School of Medicine

\section{Feng Shen}

Shanghai Jiaotong University School of Medicine

\section{Yi Zhang ( $\triangle$ xinhuagi@sina.com )}

Shanghai Jiaotong University School of Medicine

\section{Lei-Ming Xu}

Shanghai Jiaotong University School of Medicine

\section{Research Article}

Keywords: Gastroesophageal variceal bleeding, Portal hypertension, Endoscopic sclerotherapy, Endoscopic ligation, Secondary prophylaxis

Posted Date: September 16th, 2021

DOI: https://doi.org/10.21203/rs.3.rs-855914/v1

License: (a) (1) This work is licensed under a Creative Commons Attribution 4.0 International License. Read Full License 


\section{Abstract \\ Background}

Portal hypertension $(\mathrm{PH})$ frequently gives rise to severe and life-threatening complications, including hemorrhage accompanied by the rupture of esophageal and gastric varices. In contrast to the widely studied guidelines for the management of $\mathrm{PH}$ in adults, there remains controversial about the optimal endoscopic management of variceal bleeding in children for secondary prophylaxis. The study aims to determine the efficacy and safety of endoscopic variceal ligation (EVL) and sclerotherapy (EST) to control bleeding in children in our clinical center.

\section{Methods}

The retrospective study included 21 children with gastroesophageal variceal bleeding who were treated by endoscopic variceal ligation or sclerotherapy at Xinhua Hospital, Shanghai Jiaotong University School of Medicine between January 2007 and July 2020. The treatment outcome involving short-term hemostatic rate and long-term rebleeding rate were investigated. Adverse events related to the procedures, such as esophageal ulcer, esophageal stricture, abnormal embolization, pneumonia and perforation were also observed.

\section{Results}

The 21 pediatric patients who were diagnosed as moderate to severe esophageal varices concurrent with gastric varices experienced EVL or EST successfully. Hemostasis was achieved in 45 of 47 (95.7\%) episodes of upper gastrointestinal bleeding. The mean volume of each single aliquot of cyanoacrylate injected was $0.3 \pm 0.1 \mathrm{~mL}$ (range: $0.1-0.5 \mathrm{~mL}$ ). Twenty-four patients $(75 \%)$ reach varices eradication in the EVL group with a median number of procedures before eradication of $2(1-4)$ and a median time to eradication of 3.40 months (1.10-13.33). Eleven patients (52.4\%) developed rebleeding events, with the mean duration of hemostasis being $11.1 \pm 11.6 \mathrm{mo}$ (range: 1.0-39.2 mo). No treatment-related complications, for example, distal embolism, were noted with the exception of abdominal pain in one patient (4.8\%).

\section{Conclusions}

Endoscopic variceal sclerotherapy or in combination with EVL turns out to be an effective and safe approach to treat variceal hemorrhage in children for secondary prophylaxis.

\section{Introduction}


Portal hypertension $(\mathrm{PH})$ usually occurs in chronic liver disease, but for pediatric patients, their common etiology is portal vein obstruction, such as biliary atresia, congenital hepatic fibrosis, portal cavernous formation and hepatic vein obstruction. Gastroesophageal varices secondary to portal hypertension can give rise to massive upper gastrointestinal hemorrhage with a mortality rate ranging from $5 \%$ to $19 \%$ in children.[1, 2] Although current therapeutic options including surgical procedures, endoscopic minimally invasive treatment and $\beta$-blocker have been extensively applied in adults, the optimal treatment for gastroesophageal varices of pediatric patients is still a matter of controversy.[3]

Endoscopic procedures for the treatment of variceal hemorrhage included endoscopic variceal ligation (EVL) and endoscopic sclerotherapy (EST). The latter therapy would need the aid of sclerosing agent with or without tissue adhesive agent. The above manipulations were routinely used in adult patients in accordance with patient's different conditions. Unfortunately, there is a limited evidence base to guide decision making for the management of portal hypertension in children. Generally speaking, clinically relevant pediatric studies have been slow to appear and the clinical practice varies widely between different physicians, implying that many pediatric patients receive care of suboptimal quality.

Therefore, we here provided the pediatric data collected in the endoscopic center of Xinhua hospital, Shanghai Jiaotong University School of Medicine, to indicate the efficacy and safety of endoscopic therapies, since the prevention of a sentinel variceal bleed will ultimately improve survival.

\section{Methods}

2.1 Patients. From February 2013 to March 2020, 21 consecutive pediatric patients (<12 years) with variceal bleeding manifested by hematemesis or melena who underwent endoscopic variceal ligation (EVL) or endoscopic sclerotherapy (EST) in our clinical center were retrospectively enrolled in the study. The preoperative and postoperative clinical characteristics of the recruited pediatric patients were collected and analyzed. This study was approved by the Ethics Committee at Xinhua Hospital, Shanghai Jiaotong University School of Medicine. Written informed consent was obtained from all patients who has been advised of the potential risks before the endoscopic procedure.

2.2 Endoscopic definitions. The esophageal and gastric varices can be divided into four types based on the relationship with esophageal varices, as well as by their location in the stomach according to the Sarin classification: ${ }^{[4]}$ (1) gastroesophageal varix type 1 (GOV1), as the most common type, is defined as continuous esophageal varices extending into the stomach below the cardia along the lesser curvature; (2) gastroesophageal varix type 2 (GOV2), is described as a continuation of esophageal varices extending into fundus along the greater curvature of the stomach beyond the cardia; (3) isolated gastric varices mostly located at the gastric fundus are classified as isolated gastric varices type 1 (IGV1), (4) while located around the gastric body, antrum or pylorus are classified as isolated gastric varices type 2 (IGV2). [5] 
Esophageal varices were graded according to the shape of esophageal varices and the presence or absence of red sign. ${ }^{[6]}$ Grade $\otimes$ showed linear or slightly tortuous esophageal varices without red sign; Grade $\otimes$ showed linear or slightly tortuous esophageal varices with red sign or snakelike tortuous uplift but no red sign; Grade $₫$ showed serpentine tortuous uplift with red sign or beaded, nodular or tumorous esophageal varices whether there is red sign or not.

\subsection{Details of endoscopic management for esophagogastric variceal bleeding (EGVB).}

Endoscopic procedures were performed using a forward-viewing electronic gastroscope (Olympus GIFXQ290) under general anesthesia with endotracheal intubation by 2 experienced endoscopists (Y. Zhang and LM. Xu).

EST procedure The EST treatment was based on the use of sclerosing agent and tissue adhesive agent. For gastric varices, patients were managed with a "sandwich method" by injecting tissue adhesive agent (N-butyl-a-cyanoacrylate, $0.5 \mathrm{~mL}$ / piece区Beijing Fu'aile Technology Development Co.,Ltd, China) between two injections of sclerosing agent (3\% polidocanol, $1 \mathrm{~mL} /$ piece, Shaanxi Tianyu Pharmaceutical Co., Ltd, China). The external sheath tube of the 23-gauge transparent teflon injector was gently pressed on the injection point for $3 \sim 5 \mathrm{~s}$ before pulling out the needle to prevent tissue adhesive from overflowing. In general, 0.5-2 $\mathrm{ml}$ tissue adhesive was required for gastric varices based on the diameter of variceal vein. Two or more injection point was needed for a severe gastric varix to avoid ectopic embolism of a large amount of tissue adhesive. For esophageal varices, the sclerosing agent was given intravariceally with $3 \%$ polidocanol, without the use of tissue adhesive agent. We generally injected 0.5 to $1.5 \mathrm{~mL}$ of sclerosant per puncture based on the size of the varix, with a total of 6 to $10 \mathrm{~mL}$. One to 3 injections were given in each variceal column starting just above the gastroesophageal junction then proximally at 2-cm intervals. Sclerotherapy was received every 21 days until the varices were obliterated completely and no variceal column was seen.

EVL procedure EVL was performed with five or six multiband ligator device (Cook Medical, Limerick, Ireland) that consisted of transparent "Opti-Vu" barrel with preloaded latex rubber bands and attached trigger cord, multiband ligator handle, and a loading catheter. The ligation was started at or just proximal to the esophagogastric junction and extended cephalad in a slightly spiral fashion within distal $5 \mathrm{~cm}$ of the esophagus on all visible varices. At each session, each variceal cord was ligated using 1 or 2 bands. In this setting, EVL is done every 2 to 8 weeks until varices are eradicated, followed by surveillance endoscopy at 3 to 6 months after variceal eradication, and every 6 to 12 months indefinitely..

\subsection{Evaluation of therapeutic outcome. ${ }^{[7,8]}$}

Control of active bleeding was defined as complete cessation of bleeding with stable vital signs for more than 48 consecutive hours after endoscopic treatment. Variceal eradication was defined as until all visible varices are obliterated or reduced to tiny thrombosed remnants or to grade $\varangle$ size that can no longer be suctioned into the ligating device. Successful hemostasis of active gastric variceal bleeding has been defined, according to the Baveno $\mathrm{V}$ criteria as an absence of upper gastrointestinal bleeding for 
the first $120 \mathrm{~h}$ after cyanoacrylate injection. Unsuccessful hemostasis was defined as death or the need to change the therapy due to (1) fresh hematemesis or naso-gastric aspiration that removed $\geq 100 \mathrm{~mL}$ of fresh blood $\geq 2 \mathrm{~h}$ after the procedure; (2) development of hypovolemic shock; or (3) a $3 \mathrm{~g} / \mathrm{L}$ drop in hemoglobin within any $24 \mathrm{~h}$ period in the absence of transfusions) Rebleeding was defined as unsuccessful secondary prophylaxis, clinically significant rebleeding from portal hypertensive sources after day 5, or recurrent melena or hematemesis resulting in (1) hospital admission; (2) blood transfusion; (3) a $3 \mathrm{~g} / \mathrm{L}$ drop in hemoglobin concentration; or (4) death within $6 \mathrm{wk}$. Treatment failure was defined as the following: (1) failure to control index active bleeding or a rebleeding episode with 2 separate attempts by same endoscopic therapy, (2) 3 or more rebleeding episodes that required endoscopic treatment and transfusion, (3) death related to rebleeding or complication, and (4) physician's decision to change modality of treatment. Major complication was defined as any adverse event (e.g., complicated esophageal ulcer, esophageal stricture, abnormal embolization, pneumonia, pleural effusion, or perforation) that required hospitalization. A complicated esophageal ulcer was defined as treatmentinduced esophageal ulcer that was associated with bleeding, significant dysphagia, or led to the postponement of scheduled endoscopy.

\subsection{Postoperative care and follow-up}

Liquid diet for three days was suggested followed by fasting for $24 \mathrm{~h}$ after endoscopic treatment. Vital signs were closely monitored. It's required to observe whether there is bleeding, abdominal pain, chest pain, dysphagia, etc. Necessary postoperative medications for portal pressure reduction, acid suppression, infection prevention and nutritional support should be given. According to the improvement of varicose veins, guide patients to decide whether to take endoscopic treatment again.

\section{Results}

\subsection{Clinical presentation and laboratory features.}

21 pediatric patients with concomitant gastroesophageal varices received endoscopic hemostasis. The clinical characteristics of children involving 10 boys and 11 girls were presented in Table 1 . The median age was $5.5 \pm 3.0$ (range: $1-11$ years), 6 of which were under 3 years. Etiology of portal hypertension includes cavernous degeneration of portal vein (CTPV) in 17 patients (81.0\%), biliary atresia in 1 patient (4.8\%), congenital hepatic fibrosis in 1 patient (4.8\%), liver fibrosis due to Caroli's disease and Niemannpick's disease in 1 patient (4.8\%). 20 patients (95.2\%) can be categorized into GOV1 according to Sarin classification of gastroesophageal varices. The remaining 1 patient was diagnosed as IGV1. To evaluate

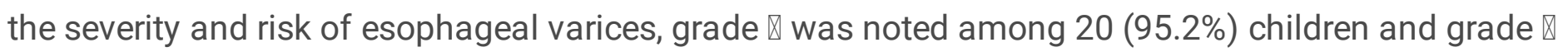
was existed in 1 child (4.8\%). The mean pediatric end-stage liver disease (PELD) score for patients younger than 12 years was $-7.2 \pm 4.69$. The average platelet count was $125.1 \pm 96.5$, which could be indicated as a noninvasive factor to predict the presence of esophageal varices in children suffering from portal hypertension. 6 among 21 pediatric patients (28.6\%) have accepted related surgery to relieve portal 
hypertension prior to endoscopic treatment, including Kasai procedure or portojejunal anastomosis, splenectomy, liver transplant, and Rex shunt.

\subsection{Outcome and Complications}

Active gastric variceal bleeding (visible bleeding or clotted blood over a gastric varix) was confirmed within $24 \mathrm{~h}$ using diagnostic esophagogastroscopy, in addition to the presence of large gastric varices and other sources of bleeding that are often present with hematemesis and melena. All patients received EST or EVL for esophageal varix and sequential application of sclerosant and tissue adhesive for gastric varix as the secondary prophylaxis. Twenty-one patients underwent endotherapy (EST $n=13, E V L n=8$ ) showed $61.9 \%$ primary eradication of varices after $1.4 \pm 0.8$ sessions. To obliterate the concomitant gastric varix, the mean injection volume of single aliquot of polidocanol was $9.1 \pm 1.2 \mathrm{~mL}$ and cyanoacrylate was $0.6 \pm 0.1 \mathrm{~mL}$. The mean injection point number for each session was $1.7 \pm 0.7$. In particular, five of the younger patients $(23.8 \%)$ failed the EVL procedure due to difficulties in approach. The successful hemostasis rate could reach complete $100 \%$ for those upper gastrointestinal bleeding, which demonstrated by the increase of hemoglobin and the negative fecal occult blood. During the follow-up duration of 2.6-90.4 mo, 8/21 (38.1\%) cases with recurred rebleeding had to perform endoscopic therapy. Rebleeding within 12 mo developed in 3/21 (14.3\%) patients.

There were no acute endoscopy-related complications noted during endoscopic therapy. But treatmentrelated complications developed in two patients $(9.5 \%)$ later, whose hospitalization had to be extended because of esophageal stricture and esophageal ulcer. Other complications, such as treatmentassociated infection, perforation, and distant emboli, did not occur. The patient aged 10 had to accept repeated endoscopic stent placement and endoscopic balloon dilatation due to esophageal stricture, then got better eventually.

\section{Discussion}

Variceal bleeding is a major complication of portal hypertension secondary to decompensated cirrhosis or portal vein obstruction, which is associated with significant mortality. Fortunately, the mortality of children with the first variceal bleed is relatively low, partly because underlying liver disease may be wellcompensated at onset of bleeding. Furthermore, comorbidities typically seen in adults were usually absent in children. That might due to the cause of portal hypertension in children is completely different from that in adults. Children were always with extrahepatic portal hypertension and the liver's function was normal, while most adult patients suffered from hepatic fibrosis and cirrhosis with severe jaundice or ascites. In our study, the majority of pediatric patients presented well-preserved liver function, since $81 \%$ of portal hypertension is indicative of cavernous degeneration of portal vein with unexplained primary causes. Moreover, the esophageal and gastric varices occur simultaneously in almost all children of our cohort. Grade 3 varices and grade 2 varices with esophageal reddish spots have been reported to be independent risk factors for EGVB. The management of esophageal and gastric varix matters for 
prevention of variceal bleeding. After the successful sequential endoscopic treatments, their living condition was always good and could grow up in a normal life.

However, patients surviving a variceal bleeding episode have very high risk of rebleeding, which is associated with mortality as high as that of the sentinel bleeding. In view of the persistence of chronic pathological process and continual progress of portal pressure, it's crucial to seek efficient means to control acute bleeding and prevent from rebleeding due to EVGB timely and effectively. With the rapid development of medical endoscopic technology, endoscopic treatment of EGVB has been a mainstay of long-term management of EGVB with significantly reduced mortality rate. However, there is no consensus on how to determine optimal endoscopic treatment for the pediatric patients.

Endoscopic variceal ligation (EVL) has been demonstrated to be effective and safe as first-line treatment to prevent or control esophageal variceal bleeding in adults and children.[5, 9] Compared to endoscopic sclerotherapy (EST), EVL is considered to bring about variceal eradication with lower number of sessions and lesser recurrence rate. However, EVL is often not suitable to manage small children because conventional banding devices has not developed for the thin esophageal lumen of children, which potentiates the esophageal injury from overtubes. ${ }^{[10]}$ Moreover, the device tip often does not reach the bleeding loci due to the angled position of the fundus in children. The Baveno $\mathrm{V}$ consensus suggests endoscopic variceal obliteration with tissue adhesive agent, for instance, N-butyl-2-cyanoacrylate (histoacryl) and EVL as first-line treatments for gastroesophageal varices (GOV) and for isolated gastric varices in adults. ${ }^{[10]}$ Despite endoscopic variceal obliteration with cyanoacrylate being more applicable to small children, caution is needed prior to its use for gastric varices in children. Fatal complications, such as systemic embolism, have been reported in patients treated with cyanoacrylate for variceal bleeding. ${ }^{[11]}$ In addition, the natural course and anatomy of gastric varices were only recently understood due to the development of interventional radiological procedures higher for gastric than for esophageal varices.

The risks of endoscopic treatment in children may exceed those in adults. These uncommonly performed procedures in small patients require general anesthesia, multiple procedures, post-operative management and a 3-day hospital admission. The current guidelines strongly recommend endoscopic ligation treatment for acute bleeding and esophageal variceal eradication based on high quality evidence. Compared with EST, EVL indeed presents a low rate of severe events, especially esophageal stenosis, which has been found in one of our cases received EST. EST occludes the veins through destroying endothelial cells and producing aseptic chemical inflammation which may subsequently results in fibrous hyperplasia of esophageal wall. However, some patients required repeated endoscopic treatment to achieve ultimate elimination of EVGB. It sometimes appears to be unwise to apply EVL since the formation of esophageal scar or the exposure of mild varicosity. Besides, EVL demonstrates its limitation of application in younger children as a result of a device entry failure. Particularly for young children under three with esophageal varices, EVL is not recommended since the banding devices are not feasible for tiny esophageal lumen. Also, there are difficulties to perform EVL for reticular communicating vessels and those post-treatment residual varix with scar. The low rate of postoperative ulcer may benefit from the intravenous injection precisely of tissue adhesive and the temporary oppression before removing the 
needle. The decreased overflow of tissue adhesive can achieve obliteration of varix well and avoid local mucosa from necrosis. Also, we try to reduce the use of tissue adhesive and decrease the unnecessary submucosal accumulation.

Since the principal problem of persistently progressive portal hypertension was not easily solved, longterm and repeated endoscopic surveillance and effective management to prevent recurrent variceal bleeding is necessary. However, this study was limited by its inclusion of a small number of patients. The efficacy and safety of tissue adhesives, such as N-butyl-2-cyanoacrylate and 2-octyl-cyanoacrylate, for acute gastric variceal bleeding require validation in large, multicenter RCTs. There is a limited evidence base to guide decision making for the management of portal hypertension in children and new clinical relevant pediatric studies have been slow to appear. Clinical practice varies widely between different physicians, implying that many patients receive care of suboptimal quality.

\section{Conclusions}

This study suggests that endoscopic vascular obliteration using the treatment of EVL and EST may be effective in children for the initial hemostasis of bleeding due to gastric varices, with a low rate of adverse events.

\section{Declarations}

\section{Ethics approval and consent to participate}

This study was approved by the Ethics Committee at Xinhua Hospital, Shanghai Jiaotong University School of Medicine. Written informed consent was obtained from all patients who had been advised of the potential risks before the endoscopic procedure.

\section{Consent for publication}

Not applicable.

\section{Availability of data and materials}

All data generated or analysed during this study are included in this published article.

\section{Competing interests}

The authors declare that they have no competing interests. 


\section{Funding}

This study was supported by the National Natural Science Foundation of China (81472844 and 82003277) and Shanghai Municipal Education Commission (16411950403 and 19411951605).

\section{Authors' contributions}

Li MM and Sun F contributed equally to the manuscript. Li MM and Sun F drafted the manuscript and analyzed the data; designed the study and supervised its implementation; Endoscopic procedures were performed by 2 experienced endoscopists (Xu LM and Zhang Y). Huai MX, Qu CY, and Shen F participated in the experiments; all authors made critical revisions and approved the final version to be published.

\section{Acknowledgements}

Not applicable.

\section{Authors' information}

Author list: Ming-Ming Li ${ }^{\#}$, Fang Sun\#, Man-Xiu Huai, Chun-Ying Qu, Feng Shen, Yi Zhang*, Lei-Ming Xu*

Li MM and Sun F contributed equally to the manuscript. Zhang $\mathrm{Y}$ and Xu LM worked as co-corresponding authors.

Institution: Department of Gastroenterology, Xinhua Hospital, Shanghai Jiaotong University School of Medicine, Shanghai, China

\section{Corresponding author information:}

Yi Zhang, MD, Department of Gastroenterology, Xinhua Hospital, Shanghai Jiaotong University School of Medicine, 1665 Kongjiang Road, Shanghai, China, 200092; Tel/fax +86 2125078999 Email: zhangyi02@xinhuamed.com.cn 
Lei-Ming Xu, MD, Department of Gastroenterology, Xinhua Hospital, Shanghai Jiaotong University School of Medicine, 1665 Kongjiang Road, Shanghai, China, 200092; Tel/fax +86 2125078999 Email: xuleiming@xinhuamed.com.cn

\section{Footnotes}

Not applicable.

\section{Abbreviations}

PH: portal hypertension

EVL: endoscopic variceal ligation

EST: endoscopic sclerotherapy

GOV1: gastroesophageal varix type 1

GOV2: gastroesophageal varix type 2

IGV1: isolated gastric varices type 1

IGV2: isolated gastric varices type 2

EGVB: esophagogastric variceal bleeding

CTPV: cavernous degeneration of portal vein

PELD: pediatric end-stage liver disease

\section{References}

1. Ling SC, Walters T, McKiernan PJ, Schwarz KB, Garcia-Tsao G, Shneider BL: Primary prophylaxis of variceal hemorrhage in children with portal hypertension: a framework for future research. Journal of pediatric gastroenterology and nutrition 2011, 52(3):254-261.

2. Maksoud-Filho JG, Gonçalves ME, Cardoso SR, Gibelli NE, Tannuri U: Long-term follow-up of children with extrahepatic portal vein obstruction: impact of an endoscopic sclerotherapy program on bleeding episodes, hepatic function, hypersplenism, and mortality. Journal of pediatric surgery 2009, 44(10):1877-1883. 
3. Shneider BL, de Ville de Goyet J, Leung DH, Srivastava A, Ling SC, Duché M, McKiernan P, Superina R, Squires RH, Bosch $\mathrm{J}$ et al: Primary prophylaxis of variceal bleeding in children and the role of MesoRex Bypass: Summary of the Baveno VI Pediatric Satellite Symposium. Hepatology (Baltimore, Md) 2016, 63(4):1368-1380.

4. Bandika VL, Goddard EA, De Lacey RD, Brown RA: Endoscopic injection sclerotherapy for bleeding varices in children with intrahepatic and extrahepatic portal venous obstruction: benefit of injection tract embolisation. South African medical journal = Suid-Afrikaanse tydskrif vir geneeskunde 2012, 102(11 Pt 2):884-887.

5. Zargar SA, Javid G, Khan BA, Yattoo GN, Shah AH, Gulzar GM, Singh J, Rehman BU, Din Z: Endoscopic ligation compared with sclerotherapy for bleeding esophageal varices in children with extrahepatic portal venous obstruction. Hepatology (Baltimore, Md) 2002, 36(3):666-672.

6. Duché M, Ducot B, Tournay E, Fabre M, Cohen J, Jacquemin E, Bernard O: Prognostic value of endoscopy in children with biliary atresia at risk for early development of varices and bleeding. Gastroenterology 2010, 139(6):1952-1960.

7. Dilawari JB, Chawla YK, Ramesh GN, Mitra SK, Walia BN: Endoscopic sclerotherapy in children. Journal of gastroenterology and hepatology 1989, 4(2):155-160.

8. Hill ID, Bowie MD: Endoscopic sclerotherapy for control of bleeding varices in children. The American journal of gastroenterology 1991, 86(4):472-476.

9. Galand J, Ley D, Coopman S, Michaud L, Guimber D, Turck D, Gottrand F: Primary prophylaxis of oesophageal variceal bleeding in children by ligation is safe and as efficient as secondary prophylaxis. Journal of hepatology 2018, 68(3):600-601.

10. Shneider BL, Bosch J, de Franchis R, Emre SH, Groszmann RJ, Ling SC, Lorenz JM, Squires RH, Superina RA, Thompson AE et al: Portal hypertension in children: expert pediatric opinion on the report of the Baveno v Consensus Workshop on Methodology of Diagnosis and Therapy in Portal Hypertension. Pediatric transplantation 2012, 16(5):426-437.

11. Saracco G, Giordanino C, Roberto N, Ezio D, Luca T, Caronna S, Carucci P, De Bernardi Venon W, Barletti $\mathrm{C}$, Bruno $\mathrm{M}$ et al: Fatal multiple systemic embolisms after injection of cyanoacrylate in bleeding gastric varices of a patient who was noncirrhotic but with idiopathic portal hypertension. Gastrointestinal endoscopy 2007, 65(2):345-347.

\section{Tables}

Table 1. Clinical characteristics of the 21 pediatric patients. 


\begin{tabular}{|ll|}
\hline Clinical characteristics & $\mathbf{n}=\mathbf{2 1}$ \\
\hline Male/female & $10 / 11$ \\
Age $(\mathrm{yr})$, & \\
Mean $\pm S D$ & $5.5 \pm 3.0$ \\
Range & $1-11$ \\
Etiology & \\
Cavernous degeneration of portal vein (CTPV) & $17(81.0 \%)$ \\
Biliary atresia & $1(4.8 \%)$ \\
Congenital hepatic fibrosis & $1(4.8 \%)$ \\
Caroli's disease & $1(4.8 \%)$ \\
Niemann-pick's disease & $1(4.8 \%)$ \\
Sarin classification of the gastroesophageal varices & \\
GoV1 & $20(95.2 \%)$ \\
IGV1 & $1(4.8 \%)$ \\
Grade of the esophageal varices & \\
Grade $\bigotimes$ & $3(14.3 \%)$ \\
Grade $\bigotimes$ & $18(85.7 \%)$ \\
Platelet counts, mean $\pm S D$ & $125.1 \pm 96.5$ \\
PELD score (<12 yr), mean $\pm S D$ & $-7.2 \pm 4.69$ \\
Related-surgery history & $6 / 21(28.6 \%)$ \\
\hline
\end{tabular}

PELD: Pediatric end-stage liver disease; NOTE. Data are expressed as mean \pm SD.

Table 2. Details of endoscopic therapy 


\begin{tabular}{|ll|}
\hline Treatment Details & $\mathbf{n}=\mathbf{2 1}$ \\
\hline Esophageal Varices & \\
EST & $13(61.9 \%)$ \\
EVL & $8(38.1 \%)$ \\
Gastric Varices & \\
Mean single aliquot of polidocanol $(\mathrm{mL})$ & $9.1 \pm 1.2$ \\
Mean single aliquot of cyanoacrylate $(\mathrm{mL})$ & $0.6 \pm 0.1$ \\
Mean injection point for each session & $1.7 \pm 0.7$ \\
Number of Sessions & $1.4 \pm 0.8$ \\
\hline
\end{tabular}

Table 3. Treatment outcome and complications

\begin{tabular}{|ll|}
\hline Treatment outcome & $\mathbf{n = 2 1}$ \\
\hline Primary Varix Eradication & $13 / 21(61.9 \%)$ \\
Hemostasis & $21 / 21(100 \%)$ \\
Follow-up duration (mo) & $2.6-90.4$ \\
Duration of hemostasis (mo) & $33.4 \pm 27.6$ \\
Rebleeding (\%) & $8 / 21(38.1 \%)$ \\
Rebleeding within 12 mo & $3 / 21(14.3 \%)$ \\
Complications & $2 / 21(9.5 \%)$ \\
\hline
\end{tabular}

NOTE. Data are expressed as mean \pm SD. 